\title{
Prolonged hyperreactivity of septal rats with intraventricular pretreatment of 6-hydroxydopamine
}

\author{
SATOSHI KURUMIYA and MAMORU UMEMOTO \\ Osaka City University, Osaka 558, Japan
}

\begin{abstract}
Fifty-four male rats were divided into four groups according to the pretreatment of 6-hydroxydopamine $(250 \mu \mathrm{g} \times 2)$ and septal lesions. The magnitude of reactivity to sensory stimulation was estimated by a 4-point rating scale. The rats that received septal lesions without 6-hydroxydopamine showed a marked increase in reactivity on the first day after surgery but a day-by-day decline in their hyperreactivity. On the other hand, the increased reactivity in 6-hydroxydopamine-pretreated septal rats was maintained over the entire course of the testing period, 130 days. Brain monoamine concentrations were assayed 20 weeks after the first injection of 6hydroxydopamine. Norepinephrine in whole brain was depleted to $13.1 \%$ of control level, dopamine decreased to $57.9 \%$ of control, but 5-hydroxytryptamine was not affected. The results indicate that long-lasting brain catecholamine depletion prolongs septal hyperreactivity in the rat.
\end{abstract}

The hypothalamus, in particular the ventromedial subdivision, plays a crucial role in mediating some emotional behaviors, and the amygdala seems to modulate hypothalamic activities. But the function of the septum, which is anatomically located between the hypothalamus and the amygdala, is not yet fully understood with regard to emotional behavior.

The involvement of the septum in emotional behavior was first demonstrated by Fulton and Ingraham (1929), who reported an increase in emotional reactivity following bilateral septal lesions in rats. Their results showed an increase in flight or attack behavior in response to formerly neutral stimuli immediately following the destruction of septal areas. Characteristic of this septal hyperreactivity are the facts that its magnitude diminishes with time and the rate of decrease in magnitude is significantly influenced by the handling of the treated animal (Brady \& Nauta, 1953, 1955; Reynolds, 1965). With daily handling, septal hyperreactivity subsided within a week, whereas with infrequent handling rats required more than $\mathbf{3 0}$ days to return to a normal state of reactivity. However, it is not clear what the effect of this handling is at the neurochemical level.

Bernard, Berchek, and Yutzey (1975) reported significant reductions in norepinephrine (NE) and dopamine (DA) levels in whole brain $48 \mathrm{~h}$ after septal lesions. But over a longer time course, Montgomery and Christian (1973) found near preoperative NE levels in whole brain 6 days after septal lesions. These findings indicate a time dependence of the NE con-

Requests for reprints should be addressed to $M$. Umemoto, Faculty of Letters, Osaka City University, Sugimoto, Sumiyoshiku, Osaka 558, Japan. centration roughly correlated with that of septal hyperreactivity.

If catecholamine (CA) is implicated in the hyperreactivity of septal rats, chronic depletion of CA prior to lesioning should delay recovery. It is well known that, through selective terminal destruction, intraventricular injection of 6-hydroxydopamine (6-OHDA) in adult animals chronically depletes CA levels in various parts of the brain (Breese \& Trayler, 1970; Uretsky \& Iversen, 1970). It might be expected that pretreatment of septal rats with 6-OHDA would prolong septal hyperreactivity by keeping catecholamine concentrations artificially low. The present study was carried out in order to determine whether this was the case.

\section{METHOD}

\begin{abstract}
Animals
Fifty-four male Wistar rats, weighing $250-300 \mathrm{~g}$, were used. The animals were housed individually and permitted free access to food and water throughout the experiment.

A cannula (26-ga stainless steel tube) was implanted under stereotaxic guidance in the lateral ventricle while the animal was anesthetized with sodium pentobarbital $(50 \mathrm{mg} / \mathrm{kg}$, ip). After 3 days of recovery from surgery, each animal was given two $20-\mu \mathrm{l}$ doses of $.9 \%$ saline vehicle solution in succession, $24 \mathrm{~h}$ apart. The volume and concentration of injected solution was determined by the results of Breese and Traylor (1970) and Uretsky and Iversen (1970). Of the animals injected, 32 received $250 \mu \mathrm{g}$ of 6-OHDA in the vehicle solution into the lateral ventricle and 22 received vehicle only. Thirty minutes prior to the first 6-OHDA injection, each animal received pargyline $(50 \mathrm{mg} / \mathrm{kg}$, ip). No treatment was given prior to the second 6-OHDA injection.
\end{abstract}

\section{Electrolytic Injury of Septal Area}

Ten days after the first injection of 6-OHDA or the vehicle solution, 31 rats (18 of the 32 6-OHDA treated rats and 13 of the 22 vehicle treated rats) received septal surgery under stereotaxic 
guidance during pentobarbital anesthesia $(50 \mathrm{mg} / \mathrm{kg}$, ip). A bipolar electrode made of two twisted stainless steel wires, each $200 \mu \mathrm{m}$ in diameter, were positioned bilaterally at points $1.0 \mathrm{~mm}$ anterior to bregma, $.5 \mathrm{~mm}$ lateral to midline of the skull, and $5.0 \mathrm{~mm}$ below dura. Direct current (about $3.0 \mathrm{~mA}$ ) was passed for $45 \mathrm{sec}$. The remaining 23 rats underwent the same treatment, except that the electrodes were lowered to $3.0 \mathrm{~mm}$ below dura and no current was passed.

The animals were divided into four groups according to drug and surgical treatment. Group 6-OHDA +S received two injections of 6-OHDA and then septal lesions; Group 6-OHDA + Sham received two injections of 6-OHDA followed by a sham operation; Group V $+\mathrm{S}$ was injected with two intraventricular vehicle solutions followed by bilateral septal lesions; and Group V + Sham received two vehicle solutions and the sham operation.

\section{Behavioral Test}

To evaluate changes in reactivity associated with 6-OHDA treatment and septal damage, a rating technique adapted from the method of Brady and Nauta (1953) was used. Ratings of animal reactivity were made for the following four behavioral components: (a) reaction to a forceps brought close to the snout, (b) reaction to a slight tapping on the back with the forceps, (c) resistance to capture with a gloved hand, (d) amount of vocalization during the above three tests. A 4-point rating scale was used for each of the behavioral components. A 0 rating was assigned to a rat that remained calm during testing, and a rating of 3 was assigned to a rat that exhibited a defensive, upright posture in response to the forceps (a), a jumping or flight response to the tapping (b), biting or severe resistance to capture (c), and incessant vocalization during the testing $(d)$.

Except for the day of operation when the septal lesions were made, behavioral testing was performed every day, from 3 days before injection of 6-OHDA or vehicle solution to the day when the rat was sacrificed.

Scores obtained from the four behavioral components were summed to provide a hyperreactivity index, and analyzed statistically by the Student $t$ test.

\section{Chemical Assay}

Twenty weeks after the first intraventricular injection of 6-OHDA or vehicle solution, the animals in Groups 6-OHDA $+\mathrm{S}$ and $\mathrm{V}+$ Sham were decapitated. The brains were quickly removed from the calvarium, cut into three pieces: hypothalamus including ventral part of thalamus, amygdala and piriform cortex, and the residual part of the brain excluding cerebellum. Then the sections were frozen on dry ice and weighed. It took, at most, $3 \mathrm{~min}$ from the time the animals were sacrificed until the time the tissues were frozen. These tissue samples were stored at $-30^{\circ} \mathrm{C}$ until assay, which always took place within 1 week. The concentrations of three monoamines in each of the brain sections were determined by the method of Karasawa et al. (1975).

\section{RESULTS}

\section{Behavioral Changes After 6-OHDA Alone}

The first treatment of 6-OHDA strongly inhibited spontaneous motor activity in the rats. All of the treated rats crouched in a dark corner of their home cages for 2 to $5 \mathrm{~h}$ after injection. Some of the animals jumped abruptly and squeaked loudly several times. On the next day, when the second 6-OHDA was given, it was not possible to discriminate between drug- and vehicle-treated rats in their spontaneous behavior. The second 6-OHDA revealed no acute effect on behavior in any of the animals.
There were no significant differences in rating scores between the two groups for 10 days until septal surgery.

\section{Hyperreactivity Caused by Septal Lesions in 6-OHDA Pretreated Rats}

Figure 1 shows the mean rating scores in all four groups on pre- and postoperative days. A marked increase in rating scores was obtained in two septal lesioned groups, 6-OHDA $+\mathrm{S}$ and $\mathrm{V}+\mathrm{S}$. On the first 2 days after septal surgery, all rats in both groups showed a marked change in reactivity in response to the extended forceps. These rats took a defensive upright posture, vocalized strongly, or demonstrated attack behavior, grasping the forceps with their forelimbs and biting it when it was brought close to the snout. Most rats jumped explosively when their backs were tapped lightly by the forceps. Additionally, the rats in Group 6-OHDA + S showed a kind of cataleptic syndrome of behavior. Once they took the upright posture in response to the presented forceps, they kept the same posture for at least $1 \mathrm{~min}$, even after the stimulus was removed. This characteristic feature of response was not observed among any of the rats in Group V + S.

The hyperreactivity of animals in Group 6-OHDA $+S$ persisted over the entire course of testing. No significant differences were found between daily postoperative scores within Group 6-OHDA + S. On the other hand, the hyperreactivity in Group $\mathrm{V}+\mathrm{S}$ was gradually attenuated. Although there were significant differences in rating scores between Group $\mathrm{V}+\mathrm{S}$ and Group V + Sham by the fifth postoperative day, from the sixth day on, no significant differences were found in rating scores between these two groups.

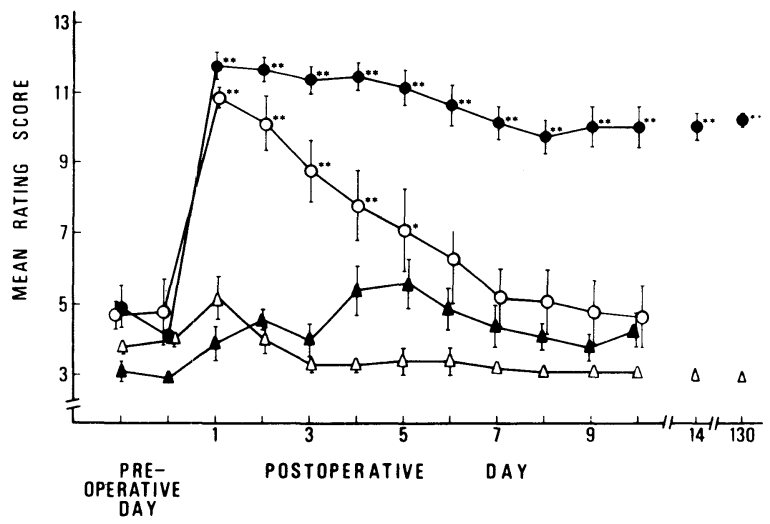

Figure 1. Mean rating score ( \pm S.E.M.) of reactivity on pre- and postoperative days in four groups. Filled circles are Group 6OHDA + S, open circles are Group $V+S$, filled triangles are Group 6-OHDA + Sham, and open triangles are Group V + Sham. Animals in Groups 6-OHDA $+S$ and 6-OHDA + Sham were administered 6-OHDA $(250 \mu \mathrm{g} \times 2)$ prior to the surgical operation. ${ }^{*} p<.05,{ }^{* *} p<.01$ vs. $V+$ Sham group by $t$ test. 
Some rats in Group 6-OHDA + Sham appeared to show a slight increase in reactivity to tactile stimulation. However, no significant differences were found in rating scores between 6-OHDA + Sham and V + Sham over the postoperative testing days.

\section{Brain Monoamine Levels in Groups 6-OHDA + S and $\mathrm{V}+$ Sham}

Table 1 shows the results of brain monoamine concentrations 20 weeks after 6-OHDA. A significant depletion of NE and DA in whole brain was seen in the animals in Group 6-OHDA $+\mathrm{S}$ in comparison with the concentrations of CA in the animals in the control group, $\mathrm{V}+$ Sham. NE level in whole brain was depleted to $13.1 \%$ of control level, and DA decreased to $57.9 \%$ of the control level. The concentration of whole-brain 5-hydroxytryptamine (5-HT), on the other hand, was not different between the two groups.

As to the results of discrete brain areas, NE depletion in amygdala and diencephalon was profound compared with DA depletion in these areas. No differences were found in 5-HT level in the three brain areas studied.

\section{DISCUSSION}

The major finding obtained in the present study was that the pretreatment of 6-OHDA prolonged the hyperreactivity induced by bilateral septal lesions in the rat.

Septal rats without 6-OHDA showed a marked increase in reactivity to visual and tactile stimulations from the first day after surgery, and they gradually lost their hyperreactivity to the stimulations with repeated daily testing. There were no statistically significant differences between the mean rating scores of the septal group and those of the sham-operated group $(\mathrm{V}+$ Sham) after the fifth postoperative day in this experiment. Septal rats with 6-OHDA, on the other hand, continued to show high reactivity scores throughout the 130 test days.

In this study, any behavioral changes in rats were not found with 6-OHDA treatment per se. This find- ing agrees with the results of other studies (Burkard, Jalfre, \& Blum, 1969; Evetts, Uretsky, Iversen, \& Iversen, 1970; Laverty \& Tayler, 1970). Nakamura and Thoenen (1972), however, observed hyperirritability after intraventricular injections of 6-OHDA in rats. Coscina, Seggie, Godse, and Stancer (1973) also reported that rage reaction was seen with single intracisternal 6-OHDA treatment. The rage reaction was maximal on the ninth day after injection and gradually diminished with daily handling. These results do not agree with ours. In order to examine the reproducibility of the results obtained in this experiment, 6-OHDA was given to another group of seven naive rats, and the result was the same-no detectable changes were found in these 6-OHDA treated rats. It is not clear why the discrepancy occurred. It should be pointed out, however, that in the daily behavioral testing in this study, we first brought the forceps close to the snout of the rat and then gave the tactile stimulation, tapping the back of the animal slightly with the forceps, since the septal rat had been known to be sensitive not only to tactile stimulation but also to visual stimulation (Brady \& Nauta, 1953; Fried, 1973). Coscina et al. (1973) and Nakamura and Thoenen (1972) did not employ visual threats in their behavioral testing.

The nuclei of the septum send efferent fibers to the vasolateral portion of the amygdala (Nauta, 1958; Powell, 1963; Swanson \& Cowan, 1979). Electrical stimulation of the septum (Siegel \& Skog, 1970) and the amygdala (Egger \& Flynn, 1963) increases the latencies of hypothalamically induced attack behavior in the cat. These results suggest that the septum has a regulating function on aggressive behavior.

The results of monoamine concentration assayed 20 weeks after the first injection of 6-OHDA showed that NE and DA levels significantly decreased in the whole brain as well as in diencephalon and amygdala. 5-HT was not affected in all discrete brain areas assayed. The septal lesions per se decreased NE and DA levels in the brain $48 \mathrm{~h}$ after surgery (Bernard et al., 1975), but this effect was not observed 7 days after surgery (Montgomery \& Christian, 1973). NE

Table 1

Effects of 6-OHDA (250 Micrograms $\times$ 2) on Brain Monoamine Concentrations

Brain Monoamine Concentrations (in Nanograms per Gram of Tissue)

\begin{tabular}{|c|c|c|c|c|c|c|c|c|c|c|c|c|c|c|c|}
\hline \multirow[b]{4}{*}{ Brain Area } & \multirow{2}{*}{\multicolumn{6}{|c|}{ Control "V + Sham" }} & \multirow{2}{*}{\multicolumn{9}{|c|}{ "6-OHDA + S" }} \\
\hline & & & & & & & & & & & & & & & \\
\hline & \multicolumn{2}{|c|}{$\mathrm{NE}$} & \multicolumn{2}{|c|}{ DA } & \multicolumn{2}{|c|}{$5-\mathrm{HT}$} & \multicolumn{3}{|c|}{$\mathrm{NE}$} & \multicolumn{3}{|c|}{ DA } & \multicolumn{3}{|c|}{ 5-HT } \\
\hline & Mean & SEM & Mean & SEM & Mean & SEM & Mean & SEM & PC & Mean & SEM & PC & Mean' & SEM & PC \\
\hline Diencephalon & 982 & 14 & 505 & 7 & 833 & 103 & 193 & 35 & 19.7 & 162 & 10 & 32.1 & 757 & 41 & 90.9 \\
\hline Amygdala & 419 & 33 & 713 & 124 & 636 & 58 & 60 & 17 & 14.3 & 479 & 43 & 67.2 & 620 & 57 & 97.5 \\
\hline Residual Parts of the Brain & 390 & 34 & 1045 & 52 & 488 & 30 & 41 & 7 & 10.5 & 631 & 30 & 60.4 & 476 & 56 & 97.5 \\
\hline Whole Brain & 472 & 104 & 935 & 46 & 542 & 31 & 62 & 7 & 13.1 & 541 & 58 & 57.9 & 575 & 62 & 106.1 \\
\hline
\end{tabular}

Note-Brain monoamines were assayed 20 weeks after the first intraventricular injection of 6-OHDA. PC = percent of controls. "6-OHDA $+S$ " animals received septal injuries 10 days after the first dose of 6-OHDA. 
and DA are supposed to be inhibitory synaptic transmitters in the brain (Szabadi, 1979). The depletion of these transmitter substances in diencephalon and amygdala is thought to elicit the disruption of septoamygdaloid influences on the hypothalamus. Fried (1973) postulated that septal hyperreactivity is induced by dysfunction in the septoamygdalohypothalamic neural circuit.

Gage and Olton (1976) reported that L-dopa, a metabolic precursor of catecholamines, reduced septal hyperreactivity. Marotta, Logan, Potegal, Glusman, and Gardner (1977) obtained similar effects with dopaminergic agonists, apomorphine, peribedil, and amphetamine. The results obtained by these recent studies are not consistent with the effects of chlorpromazine and haloperidol. These dopaminergic blockers have a transient depressant action on the septal rage syndrome (Raitt, Nelson, \& Tye, 1961; Stark \& Henderson, 1966). Marotta et al. (1977) mentioned that the effects of these agents resembled the effects exerted by such nonspecific sedatives as methohexital. They concluded that the septal syndrome was concerned with dysfunction of the dopaminergic system, and that dopaminergic agents facilitated recovery from the septal syndrome by diaschistic processes in the dopamine-rich mesolimbic system. It is conceivable that long-lasting CA depletion by 6-OHDA in this study might prevent these processes. In the biochemical assay, it was found that 6-OHDA lowered both NE and DA concentrations in the brain areas, although DA was depleted to a lesser extent than NE. Which system, NE or DA, is more crucially involved in septal hyperreactivity has to be determined in the next step.

\section{REFERENCES}

Bernard, B. K., BercheK, J. R., \& Yutzey, D. A. Alteration in brain monoaminergic functioning associated with septal lesion induced hyperreactivity. Pharmacology, Biochemistry and Behavior, 1975, 3, 121-126.

BradY, J. V., \& NaUta, W. J. H. Subcortical mechanisms in emotional behavior: Affective changes following septal forebrain lesions in the albino rat. Journal of Comparative and Physiological Psychology, 1953, 46, 339-346.

Brady, J. V., \& Nauta, W. J. H. Subcortical mechanisms in emotional behavior: The duration of affective changes following septal and habenular lesions in the albino rat. Journal of Comparative and Physiological Psychology, 1955, 48, 412-420.

BreEse, G. R., \& TraYler, T. D. Effect of 6-hydroxydopamine on brain norepinephrine and dopamine: Evidence for selective degeneration of catecholamine neurons. Journal of Pharmacology and Experimental Therapeutics, 1970, 174, 413-420.

BurKard, W. P., JAlfre, M., \& Blum, J. Effect of 6-hydroxydopamine on behavior and cerebral amine content in rats. Experientia, 1969, 25, 1295-1296.
Coscina, D. V., Seggie, J., Godse, D. D., \& Stancer, H. C. Induction of rage in rats by central injection of 6-hydroxydopamine. Pharmacology, Biochemistry and Behavior, 1973, 1, 1-6.

EgGer, M. D., \& F FYNN, J. P. Effects of electrical stimulation of the amygdala on hypothalamically elicited attack behavior in cats. Journal of Neurophysiology, 1963, 26, 705-720.

EvetTs, K. D., Uretsky, N. J., Iversen, L. L., \& Iversen, S. D. Effects of 6-hydroxydopamine on CNS catecholamines, spontaneous motor activity in rats. Nature, 1970, 225, 961-962.

Fried, P. A. The septum and hyper-reactivity: A review. British Journal of Psychology, 1973, 64, 267-275.

Fulton, J. F., \& Ingraham, F. D. Emotional disturbances following experimental lesions of the base of the brain. American Journal of Physiology, 1929, 90, 353.

GAGE, F. H., \& Olton, D. S. L-dopa reduced hyperreactivity induced by septal lesions in rats. Behavioral Biology, 1976, 17, 213-218.

Karasawa, T., Furukawa, K., Yoshida, K., \& Shimizu, M. A double column procedure for the simultaneous estimation of norepinephrine, normetanephrine, dopamine, 3-methoxytyramine and 5-hydroxytryptamine in brain tissue. Japanese Journal of Pharmacology, 1975, 25, 727-736.

LAVERTY, R., \& TAYLER, K. M. Effects of intraventricular 2,4,5trihydroxyphenylethylamine (6-hydroxydopamine) on rat behavior and catecholamine metabolism. British Journal of Pharmacology, 1970, 40, 836-846.

Marotta, R. F., Logan, N., Potegal, M., Glusman, M., \& GARDNER, E. L. Dopamine agonists induce recovery from surgically-induced septal rage. Nature, 1977, 269, 513-515.

Montgomery, R. L., \& Christian, E. L. Norepinephrine concentrations in brains and hearts of hyperreactive septally lesioned rats. Pharmacology, Biochemistry and Behavior, 1973, 1, 491492.

Nakamura, K., \& Thoenen, H. Increased irritability: A permanent behavior change induced in the rat by intraventricular administration of 6-hydroxydopamine. Psychopharmacologia, 1972, 24, 359-372.

NAUTA, W. J. H. Hippocampal projections and related neural pathways to the midbrain in the cat. Brain, 1958, 81, 319-340.

Powell, E. W. Septal efferents revealed by axonal degeneration in the rat. Experimental Neurology, 1963, 8, 406-422.

Raitt, J. R., Nelson, J. W., \& Tye, A. Effect of chlorpromazine on septal hyperactivity in the rat. British Journal of Pharmacology, 1961, 17, 473-478.

REYNOLDS, R. Equivalence of radio frequency and electrolytic lesions on producing septal rage. Psychonomic Science, 1965, 2, 35-36.

Siegel, A., \& Skog, D. Effects of electrical stimulation of the septum upon attack behavior elicited from the hypothalamus in the cat. Brain Research, 1970, 23, 371-380.

Stark, P., \& Henderson, J. K. Differentiation of classes of neurosedatives using rats with septal lesions. International Journal of Neuropharmacology, 1966, 5, 385-389.

Swanson, L. W., \& Cowan, W. M. Connections of the septal region in the rat. Journal of Comparative Neurology, 1979, 186, 621-655.

Szabadi, E. Adrenoceptors on central neurons: Micro-electrophoretic studies. Neuropharmacology, 1979, 18, 831-843.

URETSKY, N. J., \& Iversen, L. L. Effects of 6-hydroxydopamine on catecholamine containing neurons in the rat brain. Journal of Neurochemistry, 1970, 17, 269-278.

(Manuscript received January 5, 1981; revision accepted for publication September 14, 1981.) 